\title{
Swine in Biomedical Research 2014
}

\author{
by Randall S. Prather, PhD, Eric M. Walters, PhD \& Kevin D. Wells, PhD
}

\section{6-8 JULY 2014}

Sheraton Raleigh Hotel

Raleigh, NC

The Swine in Biomedical Research 2014 meeting, the fifth in a series that began in 1995 at College Park, MD, highlighted the growing utility of pig models in biomedical research. The goals of the meeting were to identify areas of study or methodologies that will enhance the utility of pigs as biomedical models; identify resource needs and areas in which new approaches or methodologies are required; stimulate interactions between researchers working with swine and with humans; identify human medical conditions more appropriately modeled by swine than by other species; and bring together individuals from agriculture and medicine to advance knowledge of pigs. There were several examples of collaboration between colleges of agriculture, of veterinary science and of medicine yielding advancements in our understanding of clinically relevant conditions.

Past meetings in the series focused on the development of tools to increase the usefulness of pig models, e.g., swine genome sequencing and genetic engineering approaches. At this meeting, however, these topics were limited to the opening session. Kyle Schachtschneider (University of Illinois, Urbana-Champaign) described the formation of a consortium to map regions of the swine genome affected by DNA methylation. Scott Fahrenkrug (Recombinetics Inc., St. Paul, MN) discussed the most recently introduced genetic engineering tools, the designed nucleases: zinc-finger nucleases (ZFNs), transcription activator-like effector nucleases (TALENs) and clustered regularly interspaced short palindromic repeats nucleases (CRISPR/cas9). Kristin Whitworth (University of Missouri, Columbia) later gave a presentation on her lab's applications of CRISPR technology to the genetic modification of pigs. The necessity of these technologies for genetic engineering of swine was supported by a talk given by R. Michael Roberts (University of Missouri, Columbia), who pointed out that although porcine induced pluripotent stem cells and embryonic-derived stem cells can develop into various cell types, they have not yet been demonstrated to contribute to the germ line. The preservation of valuable genetic resources created from these methods requires good techniques for cryopreserving gametes from pigs. One such technique was described by Ayuko Uchikura (Meiji University, Tokyo, Japan), who was able to

Prather, Walters and Wells are affiliated with the University of Missouri, Columbia, and the National Swine Resource and Research Center, Columbia, MO.

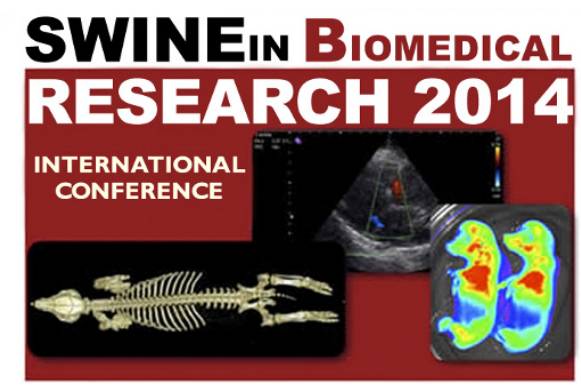

cryopreserve blastocyst-stage embryos produced in vitro that appeared morphologically normal after thawing. Bruce Whitelaw (Roslin Institute, Roslin, Scotland) discussed the widespread benefits of proposed agricultural applications for genome-editing tools, which include decreasing the environmental impact of agriculture, improving the welfare of livestock animals, decreasing antibiotic use and helping to feed the world's population.

The remainder of the meeting focused on swine models of human diseases, with speakers repeatedly noting that the pig is superior to the mouse for modeling many aspects of human physiology. Two presentations were given on the use of swine trauma models to study hemostasis. Other presentations highlighted the advantages of pigs' similar gastrointestinal anatomy and physiology to those of humans for studies of nutritional epigenetics, neonatal nutrition, intrauterine growth restriction and gastrointestinal diseases. Finally, several presentations were given on transgenic swine models of human diseases including cancer, Duchenne muscular dystrophy, cardiovascular disease, cystic fibrosis and ataxia-telangiectasia.

One of the first drivers for development of genetic engineering technologies for swine was the desire to create organs for xenotransplantation. Hiroshi Nagashima's team (Meiji University, Tokyo, Japan) genetically engineered pig embryos lacking pancreases and plan to use human stem cells to produce human pancreases in these pig embryos. They are also applying the same strategy for kidney development. David Ayares (Revivicor Inc., Blacksburg, VA) spoke about his lab's work to prevent organ rejection after xenotransplantation by using transgenes to alter some of the surface molecules on pig organs.

Several presentations were given on regenerative medicine studies using pigs. John van Aalst (University of North Carolina, Chapel Hill, NC) used swine models in preclinical studies of an approach to regenerate bone in the alveolar cleft to correct cleft pallet using umbilical cord mesenchymal stem cells. Mirek Janowski (Johns Hopkins University, Baltimore, MD) presented a method of MRI-guided delivery of labeled stem cells to the central nervous system of pigs. Two other presentations were given on the transplantation of engineered tissue scaffolds in pigs to assess host tissue integration into the scaffold. 